\title{
Optimization Of Cruise Tourist Orbit For Multiple Targets On GEO
}

\author{
Le-tian Zheng ${ }^{1,}$, Fei Feng ${ }^{1}$ and Yan-li $\mathrm{Xu}^{1}$ \\ The academy of equipment, Beijing 101416, China \\ a18641724182@163.com,
}

Keywords: Geosynchronous Orbit, Multiple Targets, Optimization, particle swarm optimization

\begin{abstract}
It is supposed to maneuver orbit if the tourist orbit is used to make detailed investigation of multiple targets on GEO. The problem is how to minimize the energy dissipations of the orbit maneuver. In this paper, the optimization strategy is offered: 1 . The difference of individual orbit inclination is supposed to be minimum by adjusting the configuration parameters such as radius of cruise, cruise speed and phase difference; 2 . The pulse position is supposed to adjust based on the achieving the task that the targets on GEO are closely observed. Finally, the results show that the proposed optimization strategy can reduce the energy consumption greatly through the two target spacecraft and multiple target spacecraft simulation.
\end{abstract}

\section{Introduction}

With the development of space technology, the development of space satellite application technology is strengthened. GEO is about $36000 \mathrm{~km}$ far from the ground and it has a unique advantage in communication, missile warning, electronic reconnaissance mission. It is because of the specialty of GEO height, the targets of cataloging and identification are very difficult ${ }^{[1]}$. It has become the focus of national development that how to observe satellites closely on GEO.

It is an observation method that has the application prospect that using tourist orbit near the multiple targets on GEO to fly-around investigate targets. But the satellites on GEO are supposed to stay in different angle of orbital plane because of the north and south position control ${ }^{[2]}$. The orbit maneuver must be implemented if each of the targets is detailed investigation. Based on how to minimize the energy dissipations of the orbital maneuver problem, in this paper, the cruise spiral configuration parameter selection and pulse moments as the design variables of optimization model are discussed. The particle swarm optimization algorithm is used to solve the optimization problem. Finally the simulation results show that the proposed optimization method can greatly reduce the energy dissipations of the orbit maneuver.

\section{Trajectory Optimization Problem Description}

There is phase difference between the observing spacecraft and the target spacecraft on GEO. Observing spacecraft is supposed to detailed investigate multiple targets in given time and orbital maneuver ability range. In this paper, through the analysis of double pulse Lambert rendezvous orbit maneuver between time and minimum energy consumption, an optimum of transfer trajectory is supposed to find to minimum energy consumption on the premise of meet the constraints and mission requirements.

\section{Lambert Problem}

The essence of Lambert problem is that the position vector of two points $P_{1} 、 P_{2}$ are $r_{1} 、 r_{2}$, and flight time $\Delta t$, strives for the orbit parameters between two points in the two-point boundary value problems. The Lagrange's form of transfer equation of time ${ }^{[3]}$ can be represented as: 


$$
\left\{\begin{array}{l}
\Delta t=\sqrt{\frac{a^{3}}{\mu}}[2 N \pi+\alpha-\beta-(\sin \alpha-\sin \beta)] \\
\cos \alpha=1-\frac{s}{a} \\
\cos \beta=1-\frac{(s-d)}{a}
\end{array}\right.
$$

In this type, $\mu$ as the earth's gravitational constant, $\alpha 、 \beta$ as the Lagrange constant, $a$ as the transfer orbit semi-major axis, $s=\frac{\left(r_{1}+r_{2}+d\right)}{2}, N$ as the transfer laps, $d=\left|r_{1}-r_{2}\right|$.I $\mathrm{n}$ this paper Newton iterative method is used to solve it to find out $v_{1}^{+}, v_{2}^{-}, \boldsymbol{v}_{1}^{-}$as the initial speed, $\boldsymbol{v}_{2}^{+}$as the terminal velocity. The total increment speed of Lambert transfer is $\Delta \boldsymbol{v}=\left|v_{1}^{+}-v_{1}^{-}\right|+\left|v_{2}^{+}-v_{2}^{-}\right| . "-", "+"$ is respectively as before change track and after change track.

\section{The Objective Function and Constraint Conditions}

Assuming that applying control for trajectory is pulse thrust. Pulse thrust assumes that: when the spacecraft change the orbit, its position vector does not change, and the velocity vector is can be expressed as:

$$
\left\{\begin{array}{c}
\boldsymbol{r}^{+}=\boldsymbol{r}^{-} \\
\boldsymbol{v}^{+}=\boldsymbol{v}^{-}+\Delta \boldsymbol{v}
\end{array}\right.
$$

In the type, $\boldsymbol{r}^{+} 、 \boldsymbol{r}^{-} 、 \boldsymbol{v}^{+} 、 \boldsymbol{v}^{-}$is respectively as the spacecraft location vector and velocity vector.

(2) Optimization of objective function

$$
\operatorname{Min} \Delta v=\sum_{i=1}^{n}\left|\Delta v_{i}\right|
$$

In the type, $\Delta \vec{v}_{i}=\vec{v}_{i}-\vec{v}_{i-1}, n$ as the number of pulses applied.

(3) Constraints

Tourist orbit is supposed to meet many constraints to detailed investigate the target on GEO, and the constraints can be summarized as:

$$
\left\{\begin{array}{l}
R_{\text {Safety }}<R<R_{\text {max }} \\
\Delta t_{\text {span }_{i}}>\Delta t_{\text {span }_{\min }} \\
T_{\text {All }}<T_{\text {Demand }}
\end{array}\right.
$$

In the type, $R$ is distance between the observing spacecraft and target spacecraft. $R_{\text {Safety }}$ is the minimum distance to avoid collisions, generally $R_{\text {Safety }}=50 \mathrm{~km} ; R_{\max }$ is the maximum distance to observe, generally $R_{\max }=200 \mathrm{~km} . \Delta t_{\text {span }_{i}}$ is single target detection time, $\Delta t_{\text {span }_{\min }}$ is minimum observation time; $T_{\text {All }}$ is total time to complete all the target observation, $T_{\text {Demand }}$ is required time of task.

(4) The optimized variables

Optimization of main variables is: $R^{[4]}$ orbit radius; $V$ orbit speed; $\phi$ the normal Angle values; $i_{0}$ initial orbit inclination, $i$ changed orbit inclination; $t_{i-1}, t_{i}(i=1, \cdots n)$ the moment of control.

\section{The Choice of Optimization Algorithm}

In recent years, the evolutionary algorithm is used to complete the pulse train problem by constructing a general optimization model ${ }^{[5]}$. The particle swarm optimization algorithm use the global search strategy optimization based on swarm intelligence. Each particle updates its speed according to the global optimal position and its optimal position. And the particle swarm optimization algorithm is more efficient than genetic algorithm in sharing information mechanism ${ }^{[6]}$. Therefore 
particle swarm optimization has faster convergence speed and global search ability in theory. And it is used to solve problem in this paper.

Particle swarm optimization algorithm is descried as follows: in $\mathrm{n}$ dimensional search space, consisting of a particle population $X=\left\{x_{1}, \ldots x_{i}, \ldots, x_{m}\right\}$, number $i$ particle position is $x_{i}=\left(x_{i 1}, x_{i 2}, \ldots, x_{i n}\right)^{T}$, and its velocity is $v_{i}=\left(v_{i 1}, v_{i 2}, \ldots, v_{i n}\right)^{T}$. Its individual extremum is $p_{i}=\left(p_{i 1}, p_{i 2}, \ldots, p_{i n}\right)^{T}$, populations of global extremum is $p_{g}=\left(p_{g 1}, p_{g 2}, \ldots, p_{g n}\right)^{T}$. Update particle velocity and position according to the following formulation:

$$
\left\{\begin{array}{l}
v_{i d}^{(t+1)}=\omega f_{i d}^{(t)}+c_{i} r_{i}\left(p_{i d}^{(t)}-x_{i d}^{(t)}\right)+c_{2} r_{2}\left(p_{g d}^{(t)}-x_{i d}^{(t)}\right) \\
x_{i d}^{(t+1)}=x_{i d}^{(t)}+v_{i d}^{(t+1)}
\end{array}\right.
$$

In the type, $d=1,2, \ldots n, \quad i=1,2, \ldots m, \quad m$ is the population size, $t$ is current Number of evolution, $r_{1}$ and $r_{2}$ is distributed in a random number between $[0,1] ; c_{1}$ and $c_{2}$ is accelerate the constant, $\omega$ known as the inertia constant. In order to ensure that the algorithm has good convergence and search ability, $\omega$ is made to change with the number of iterations of linear function. In this paper fitness function is the objective function.

\section{Optimization Idea}

There are two steps need to be optimized:

(1) The beginning orbit design

In orbit transfer problems in different planes, the change of the orbital inclination are often occupied the main part of the energy consumption. In order to reduce the consumption of energy, we first need to think about how to make the transfer orbit plane Angle difference minimal. Assumes that the target spacecraft1 is in less a relative inclination orbit, and the target spacecraft 2 is in a relatively greater inclination. The optimization of work is that minimize the difference of their orbit inclination by adjusting the configuration parameters

(2)The selection of pulse points

The choice of pulse moment in the case of transfer time fixed determines the orbit control energy and observed the effect undoubtedly. Specifically it includes the following parameters:

- The starting point location in first orbit, or the starting moment in first orbit.

- The entry point location in second orbit, or the entry moment in second orbit.

When specific optimization, firstly, ensure that the GEO targets are detailed observed in a cycle by the cruise spacecraft. And then adjust the position of the two pulse points. At the same time the optimization algorithm is used to solve the minimum energy consumption problem.

\section{Simulation Example}

\section{Double Target Detailed Observation Track Optimization}

Set two GEO targets orbital elements as shown in Table 3.1.

Table. 3.1 Orbital elements of GEO targets

\begin{tabular}{cccccc}
\hline Sat & $a(\mathrm{~km})$ & $i\left(^{\circ}\right)$ & $\Omega\left(^{\circ}\right)$ & $\omega\left(^{\circ}\right)$ & $\theta\left(^{\circ}\right)$ \\
\hline GEO_1 & 42164 & 0 & 0 & 0 & -2 \\
GEO_2 & 42164 & 2 & 0 & 0 & -4 \\
\hline
\end{tabular}

Firstly optimize the orbit inclination, parameters of particle swarm algorithm are set to: due to the design parameters are $R, V, \phi$, dimension is three, the number of particle swarm is 10 , accelerating factor: $c_{1}=c_{2}=1.496$, inertial factor $\omega=0.7$, the number of iterations is set to 100 times. The scope of Orbit radius $R$, winding speed $V$, Angle $\phi$ is shown in the Table below: 
Table 3.2 The scope of design parameters

\begin{tabular}{lccc}
\hline Design parameters & $R(\mathrm{~km})$ & $V(\mathrm{~km} / \mathrm{s})$ & $\phi\left({ }^{\circ}\right)$ \\
\hline The scope of design parameters & $100 \sim 200$ & $0.004 \sim 0.006$ & $-35 \sim 35$ \\
\hline Calculate orbit inclination of two orbits, the optimal design variable values as shown in Table3.3. \\
$\begin{array}{l}\text { The Sat_1 and Sat_2 are respectively two orbit rounds spacecraft. The two orbit inclination difference } \\
\text { and phase difference exists between the tracks are shown clearly in figure3.1 by STK. } \\
\text { Table.3.3 Optimal design variable values }\end{array}$ \\
\hline$R(\mathrm{~km})$ \\
\hline \multicolumn{4}{c}{$(\mathrm{km} / \mathrm{s})$} \\
\hline Sat_1 \\
\hline Sat_2
\end{tabular}

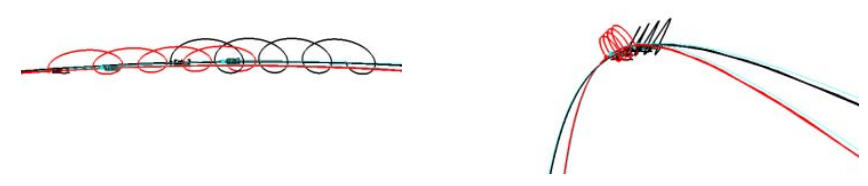

Fig 3.1 Cruise orbit as orbit inclination is optimal

Then the pulse points of transfer are computed, the optimal transfer time is $11 \mathrm{~h}$, the optimal pulse calculation results as shown in Table 3.4.

Table 3.4 energy consumption of transfer

\begin{tabular}{|c|c|c|c|c|c|c|}
\hline & No optimal & \multicolumn{3}{|c|}{ Optimal orbit inclination } & \multicolumn{2}{|c|}{$\begin{array}{l}\text { Optimal orbit inclination and } \\
\text { pulse point optimization }\end{array}$} \\
\hline$\Delta V_{\text {total }}(\mathrm{km} / \mathrm{s})$ & 0.1325 & \multicolumn{3}{|c|}{0.0977} & \multicolumn{2}{|c|}{0.0960} \\
\hline \multicolumn{7}{|c|}{$\begin{array}{l}\text { Multiple Target Detailed Observation Track Optimization } \\
\text { For three or more targets, orbit inclination should not be the only factor, phase relation of GEO } \\
\text { targets should also be considered. Do not break general, this example takes three GEO satellite as an } \\
\text { example to analyze (three satellite's orbit elements as shown in Table 3.5). The result is shown in } \\
\text { figure 3.2. } \\
\qquad \text { Table 3.5 The orbit elements of three GEO targets }\end{array}$} \\
\hline & $a_{(\mathrm{km})}$ & $e$ & $\left.i_{(}{ }^{\circ}\right)$ & $\Omega\left({ }^{g}\right)$ & $\left.\omega_{\left({ }^{\circ}\right)}\right)$ & $\theta_{(9)}$ \\
\hline GEO_1 & 42164 & 0 & 3 & 320 & 0 & 0 \\
\hline GEO_2 & 42164 & 0 & 0 & 326 & 0 & 0 \\
\hline GEO_3 & 42164 & 0 & 5 & 340 & 0 & 0 \\
\hline
\end{tabular}

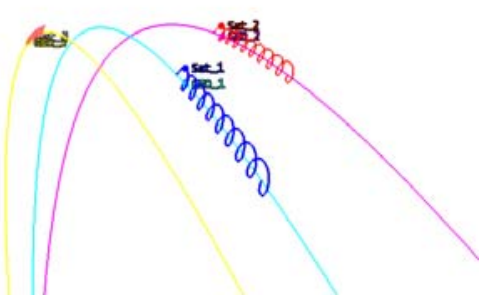

Fig 3.2 Cruise orbit of three targets

The figure 3.2 shows that the difference of orbit inclination between three targets is big, orbital 
maneuver need to consume lots of energy. Considering the difference of orbit inclination is the most important factor of energy transfer, the optimization method is:

(1)Arrange the targets according to the orbit inclination from small to large;

(2)Optimize the design of the head and tail of targets;

(3)Optimize the pulse moment.

Firstly, optimize the orbit inclination of the head and tail of targets to maximize the GEO_2 and minimize orbit inclination of GEO_3. Therefore it makes the total angle difference of transfer process minimum. Finally, the particle swarm algorithm is used to solve the problem, and the parameters setting are similar to the previous section, there is no longer here.

Then, the pulse moment is supposed to be optimized. And the dimension of particle swarm is 2 , the rest of the parameters do not change. Total transfer energy is the objective function, and the first and second pulse moments are the design variables. The optimal solution is shown in the Table 3.6

Table 3.6 The velocity increment of GEO 2 to GEO_1

\begin{tabular}{|c|c|}
\hline Pulse position(km) & velocity increment $(\mathrm{km} / \mathrm{s})$ \\
\hline 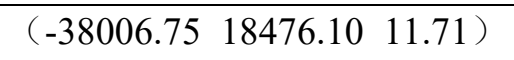 & 0.10856 \\
\hline$(29238.68-30357.50-222.25)$ & 0.14718 \\
\hline
\end{tabular}

Fig 3.3 The Transfer trajectory of GEO_2 to GEO_1

Table 3.7 The velocity increment of GEO 1 to GEO 3

\begin{tabular}{|c|c|}
\hline Pulse position(km) & velocity increment $(\mathrm{km} / \mathrm{s})$ \\
\hline$(-41887.79-4573.13-1527.59)$ & 0.3507 \\
\hline$(42149.62-1887.11 \quad 1079.17)$ & 0.3370 \\
\hline
\end{tabular}

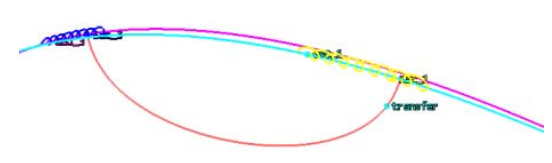

Fig 3.4 The transfer trajectory of GEO 1 to GEO 3

From Table 3.8, the energy consumption of the orbital transfer is reduced greatly after the two-optimization.

Table 3.8 The energy consumption of transfer

\begin{tabular}{ccc}
\hline & No optimal & $\begin{array}{c}\text { Optimal orbit inclination and } \\
\text { pulse point optimization }\end{array}$ \\
\hline$\Delta V_{\text {total }}(\mathrm{km} / \mathrm{s})$ & 1.4804 & 0.9434 \\
\hline
\end{tabular}




\section{Summary}

Orbit maneuver is necessary to detailed investigate multiple targets on GEO using the cruise orbit. In this paper, a optimization strategy is given based on particle swarm optimization algorithm: 1 . The difference of individual orbit inclination is supposed to be minimum by adjusting the configuration parameters such as radius of cruise, cruise speed and phase difference; 2. The pulse position is supposed to adjust based on the achieving the task that the targets on GEO are closely observed. Finally, the results show that the proposed optimization strategy can reduce the energy consumption greatly through the two target spacecraft and multiple target spacecraft simulation.

\section{References}

[1] Le-ping Yang. Spacecraft relative motion trajectory planning and control [M]. National defense industry press, 2010.

[2] Li Hengnian Gao Yijun, Yu Peijun, et al. Geostationary orbit, a control strategy study [J]. Journal of aerospace, 2009, 30 (03) : 967-973.

[3]VALLADO D A. Fundamentals of astrodynamics and applications [M] . 2nd ed. EI Segundo, California: Microcosm Press, 2001:303-395.

[4] Xu Yanli Zhang Yasheng. Cruise spiral design and simulation [C] / / system simulation technology and its application research papers. 2014.

[5]LUO Y Z, TANG G J, LEI Y J, et al. Optimization of multiple impulse multiple-revolution rendezvous phasing maneuvers $[\mathrm{J}]$. Journal of Guidance, Control and Dynamics, 2007, 30(4): 946-952.

[6]KENNEDY J, EBERHART R. Particle swarm optimization [C] / / IEEE International Conference on Neural Networks. New York: IEEE, 1995:1942-1948 